Volume 9, No.4, July - August 2020

International Journal of Advanced Trends in Computer Science and Engineering

Available Online at http://www.warse.org/IJATCSE/static/pdf/file/ijatcse47942020.pdf

https://doi.org/10.30534/ijatcse/2020/47942020

\title{
Power Spectral Density Analysis of Subsurface Electromagnetic Wave (EM) Radar Implemented in USRP 2932
}

\author{
Vrian Jay V. Ylaya \\ College of Engineering and Information Technology, Surigao State College of Technology, Philippines, \\ vylaya@ ssct.edu.ph
}

\begin{abstract}
This paper presents detection buried objects such as metal, plastic, and water using power spectral density analysis implemented in software-defined radio USRP 2932. Each of the objects is buried in sandy soil with varying distances and capture every power spectral density (PSD) value. The obtained PSD of each material decreases as the depth increases, and each material has a unique value of power spectral density (PSD), as shown by the results of experiments. Thus, subsurface electromagnetic (EM) radar detection is possible from the power spectral density analysis of reflected signals using simple pulse position modulation (PPM) implemented in software-defined radio.
\end{abstract}

Key words: Power spectral density, software-defined radio, subsurface detection, pulse-position modulation

\section{INTRODUCTION}

Subsurface electromagnetic radar is now widely used by professionals for rapid exploration of what's beneath everything without the need for unnecessary disruption and extraction [1]. The utilization of electromagnetic wave radar in the field of construction and civil engineering industries is now in-demand, where mineral resources, water, environmental aspects, and even security are the target of interest [2]. The techniques are based on the radar concept where the signal is transmitted, and the reflected signal from the target is analyzed. Reflected signals are composed of interference waves and reflected waves. Better display of geological profile one must suppress the interference wave using signal processing [3]. Reflection wave is essential for quality data for interpretation, and this depends on what signal processing is used, such as filtering, deconvolution, migration, and homing processing [4]. Fast computers are now available for mathematical computations for filtered discrete signals since records of reflected signals are in series of discrete-time series, thus digital filtering is suitable [5].
This research aimed to implement primary pulse position modulation and process the reflected signals using power spectral density (PSD) value, which will be used to interpret subsurface detection of material. Design of graphical user interface and the pulse position modulation is implemented in LabVIEW [6]. Raw data files from the LabVIEW are converted to CSV files, and power spectral density (PSD) analysis is done using MATLAB [7]. The underlying implementation of necessary pulse position modulation and power spectral density analysis can help to recognize the subsurface anomaly [8].

\section{THEORY}

\subsection{Power Spectral Density of Wide-sense stationary Random Process}

The power spectral density PSD of wide-sense stationary WSS random process $X(t)$ is given by the Fourier transform (FT) of its autocorrelation function

$$
S_{X}(f)=\int_{-\infty}^{\infty} R_{X}(\tau) e^{-j z \pi f t} d \tau
$$

$R_{X}(\tau)$ is a measure of the rate of change of $X(t)$ with time and hence is related to the frequency response of $X(t)$.

\subsection{The response of Linear-Time Invariant system to Random Signals}

Consider an LTI system $h(t)$

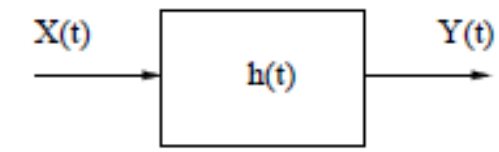

$$
\begin{aligned}
& E[Y(t)]=m x \int_{-\infty}^{m} h(\tau) d \tau=\operatorname{mx} H(0) \\
& E[Y(t) Y(t+\tau)]=\int_{-\infty}^{\infty} \int_{-\infty}^{\infty} h(r) h(s) R_{X}(\tau+s-r) d s d r
\end{aligned}
$$

Two processes $X\left(t^{\prime}\right)$ and $Y(t)$ are jointly WSS

$R_{X Y}=\int_{-\infty}^{\infty} h(s) R_{X}(\tau+s) d s=h(-\tau) * R_{X}(\tau)$ 
From these, we also obtain

$$
R_{Y}(\tau)=\int_{-\infty}^{\infty} h(r) R_{X Y}(\tau-r) d r=h(\tau): R_{X Y}(\tau)
$$

\subsection{Frequency Domain Analysis}

Taking the Fourier transforms of the correlation functions, we have

$$
\begin{gathered}
s_{X Y}(f)=H^{*}(f) s_{X}(f) \\
s_{Y}=H(f) S_{X Y}(f)
\end{gathered}
$$

where $H^{*}(f)$ is the complex conjugate of $H(f)$

The output-input power spectral density PSD relation

$$
s_{Y}(f)=\|H(f)\|^{2} s_{X}(f)
$$

Since the soil as a low pass filter[1], we can change the unit impulse response correlation function of low pass filter $\|\left. H(f)\right|^{2}$. The ideal low-pass digital filter frequency response of ideal low-pass filter is:

$$
p(w)= \begin{cases}1 & w=w_{0} \\ 0 & w>w_{0}\end{cases}
$$

So, the unit sampling function of the filter is:

$$
h(n)=\frac{\sin \left(w_{0} n\right)}{\pi n}
$$

Therefore, $h(n)$ is sinc function symmetrically based on $h(0)=\frac{w_{n}}{\pi}$. Such system is causal, and therefore it is physically impossible. However, if we intercept $(n)$, and shift it after the interception, we can get:

$$
h^{r}(n)=h\left(n-\frac{M}{2}\right) \quad n=0,1,2,3,4,5, \ldots . . M
$$

So $h^{l}(n)$ is causal and finite long. The length of it is $M+1$. Let

$$
H(z)=\sum_{n=0}^{M} h^{E}(n) z^{-n}
$$

We can get the transfer function of the designed filter. The frequency response of $H(z)$ is near $q(w)$, and it is similar to that of linear.

Analyzing the formula (2), the average output power spectral density $s_{Y}(f)$ is a convolution of input power spectral density $S_{X}(f)$ and squared of unit impulse response correlation function $|H(f)|^{2}$ hence there will be a reduction of $s_{Y}(f)$ which is also depend on the medium $|H(f)|^{2}$. When dealing with power signals, it is essential to characterize the signals with their autocorrelation function, or equivalent in the frequency domain, their power spectral density[9].

\section{METHOD}

Figure 1 shows the system and set up of the subsurface electromagnetic radar system based on software-defined radio (SDR). It consists of NI USRP 2932 device, two antennas for transmission and reception, a PC host or a laptop, and an RF amplifier. The software-defined radion (SDR) was implemented through LabVIEW and NI USRP 2932. The transmitted pulse position modulated signal was generated in LabVIEW and was up-converted to an analog signal by the NI USRP 2932.

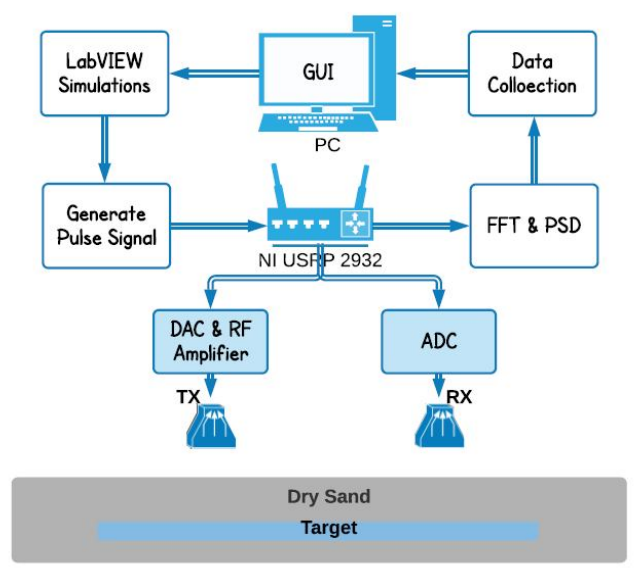

Figure 1: System and Set up

The received signal, which is a cluster of reflected signals, was transformed to its power spectral density (PSD) to obtain the critical information of the target. A graphical user interface (GUI) was provided for parameter settings and displayed for the interpretation of the real-time data.

\subsection{PPM-based Subsurface Electromagnetic Radar System Implementation}

The subsurface electromagnetic wave (EM) radar system is designed and implemented in LabVIEW, which is based on the theoretical studies and analyses of the system. The program is designed to generate a pulse position modulation that will be transmitted to the ground. Then, it will receive the reflected pulse signal and perform partial signal processing, which transforms the time-domain signal to its power spectral density.

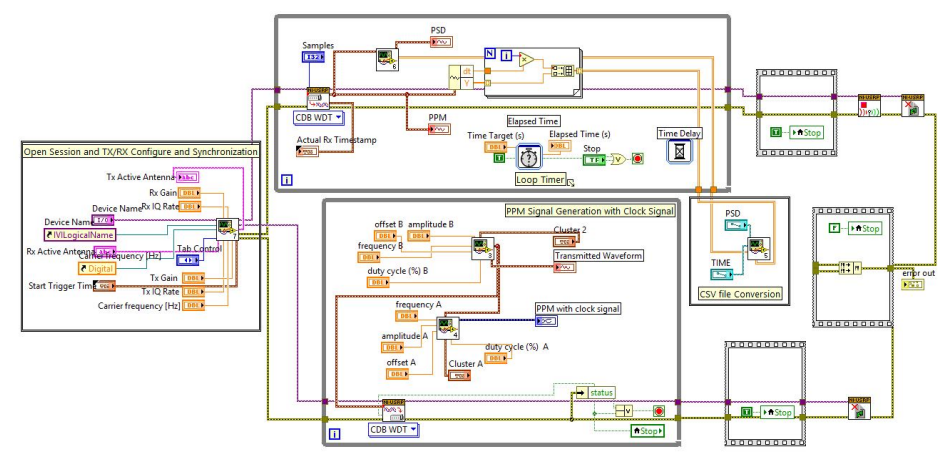

Figure 2: PPM-based subsurface electromagnetic radar system block diagram implemented in LabVIEW

Figure 2 shows the block diagram of the pulse-position modulation (PPM)-based electromagnetic wave (EM) radar system. It can be subdivided into eight blocks, in which each of them performs its specific task. These are: (1) Open Session TX/RX Configuration and Synchronization; (2) PPM Signal Generation with Clock Signal; (3) Signal Transmission; (4) Signal Reception; (5) Signal Processing; (6) Loop Timer; (7) Signal Conversion to CSV Format; (8) Close Session.

As shown in Figure 3, the pulse-position modulation (PPM) transmitted signal and in Figure 4 shows the 
pulse-position modulation (PPM) signal with modulating the carrier frequency, which will be transmitted on the antenna.

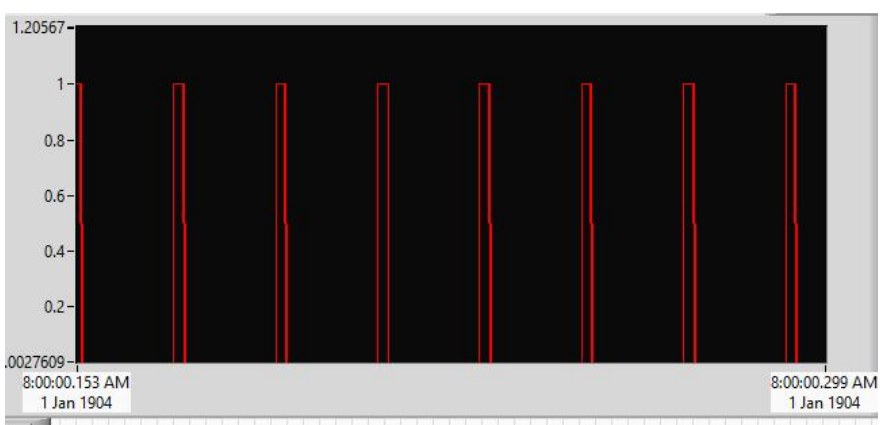

Figure 3: Pulse-position Modulation (PPM) Signal

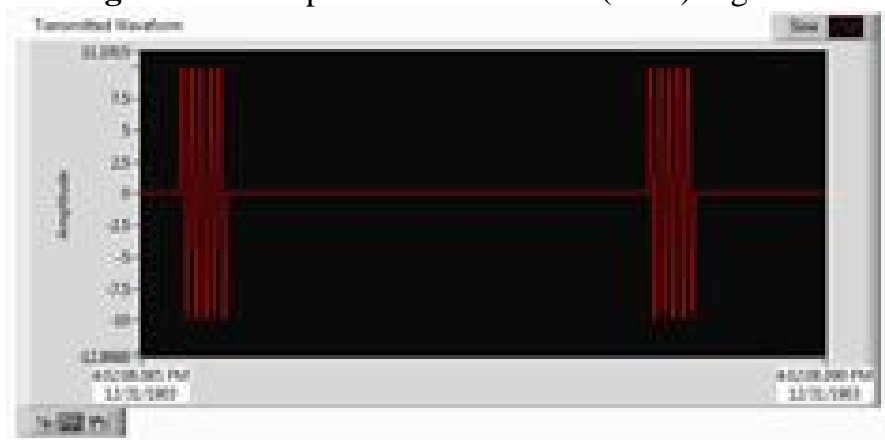

Figure 4: Transmitted Waveform

The seventh block, shown in Figure 5, writes the real-time received signal and power spectral density (PSD) signal to comma-separated values (CSV) file format through the Write Delimited Spreadsheet VI. These data will be loaded to MATLAB as inputs for further signal processing and interpretation.

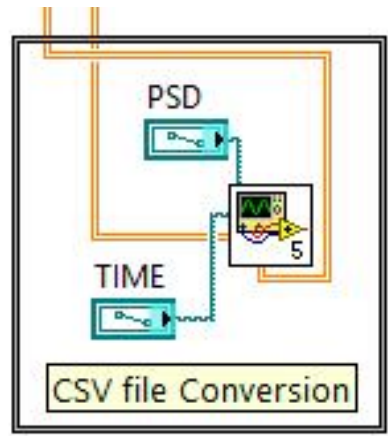

Figure 5: Exporting Signal to CSV Format

\subsection{Signal Processing in LabVIEW}

A partial signal processing of the received signal is done in LabVIEW through the Power Spectral Density (PSD) Virtual Instrument (VI), where the time-domain signal is transformed to its frequency-domain counterpart. This is dome because the vital information of the signal (e.g., power and peaks) is provided by its power density spectrum.

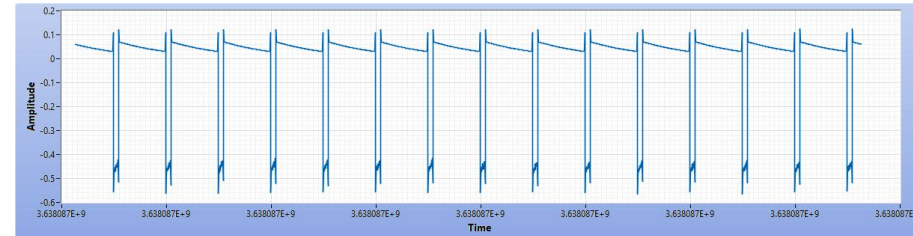

Figure 6: Received Pulse Train

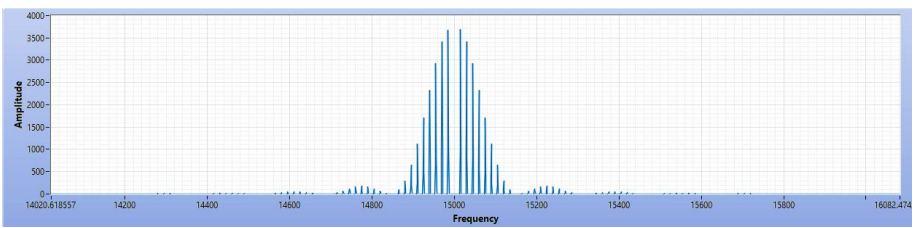

Figure 7: Power Spectral Density (PSD) Spectrum of the Received Signal

Figure 6 shows the received pulse train signal of the first sample of no target testing and its power density spectrum, as shown in Figure 7, which is a sinc function. Both signal and spectrum are sampled at 30,000 samples and are exported to comma-separated values (CSV) file format for further processing.

\subsection{System Calibration Setup}

Calibration was done to achieve consistency and the optimum power performance of the pulse position modulation (PPM) subsurface electromagnetic wave (EM) radar system. Varying the carrier frequency depending on the performance of the available antenna and the position of the antennas from the ground and between antennas separation, then observing the trend of the output of the PSD VI seen in the GUI as shown Figure 8 and Table 1 on the experimental setup. Two (2) variations were made to the carrier frequency, three (3) variations are made to the distance between the two antennas and four (4) variations to the distance of the two antennas with respect to the ground; thus, a total of twelve (12) setups were made for each carrier frequency.

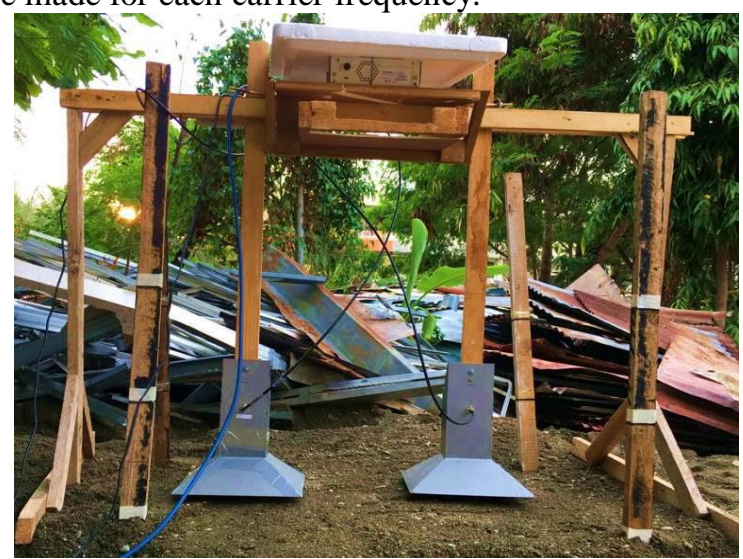

Figure 8: Experimental Setup

The depth of the buried metal was varied in each setup, listed in Table 1 to test optimum set up. The gain of the TX and RX antennas were set to $10 \mathrm{~dB}$. In each setup, fifteen (15) data samples were gathered. The average maximum values of the fifteen (15) data samples in each setup were computed and plotted. 
Table 1: Distance Information between Sep Up

\begin{tabular}{|c|c|c|}
\hline Set-up & $\begin{array}{c}\text { Distance Between } \\
\text { Antennas (m) }\end{array}$ & $\begin{array}{c}\text { Distance of Antennas } \\
\text { wrt Ground (m) }\end{array}$ \\
\hline 1 & 0.55 & 0.01 \\
\hline 2 & 0.55 & 0.10 \\
\hline 3 & 0.55 & 0.30 \\
\hline 4 & 0.55 & 0.50 \\
\hline 5 & 0.65 & 0.01 \\
\hline 6 & 0.65 & 0.10 \\
\hline 7 & 0.65 & 0.30 \\
\hline 8 & 0.65 & 0.50 \\
\hline 9 & 0.90 & 0.01 \\
\hline 10 & 0.90 & 0.10 \\
\hline 11 & 0.90 & 0.30 \\
\hline 12 & 0.90 & 0.50 \\
\hline
\end{tabular}

Table 2: Depth Variation of Metal Testing for Calibration

\begin{tabular}{|c|}
\hline Depth Tested \\
\hline no target \\
\hline $0.1 \mathrm{~m}$ \\
\hline $0.5 \mathrm{~m}$ \\
\hline $0.8 \mathrm{~m}$ \\
\hline
\end{tabular}

\subsection{Experimental Setup}

Among the twelve (12) setups, setup 1 has the best performance and the most consistent, as shown in Figure 9. The average power is decreasing as the depth of the target increases. This is true since the pulse-position modulation (PPM) radar system is susceptible to external movement and background noise, and this setup is the most stable since the antennas are very close to the ground. For the comparison between the two carrier frequencies, the change of the average power of series $2(2.2 \mathrm{GHz})$ is gradual, while series 1 (1.7 $\mathrm{GHz}$ ) is very noticeable. This is also true for subsurface detection radar that lower frequencies penetrate farther than higher frequencies. Therefore, setup 1, shown in Figure 9, with a carrier frequency of $1.7 \mathrm{GHz}$, is chosen throughout the whole experiment and data gathering.

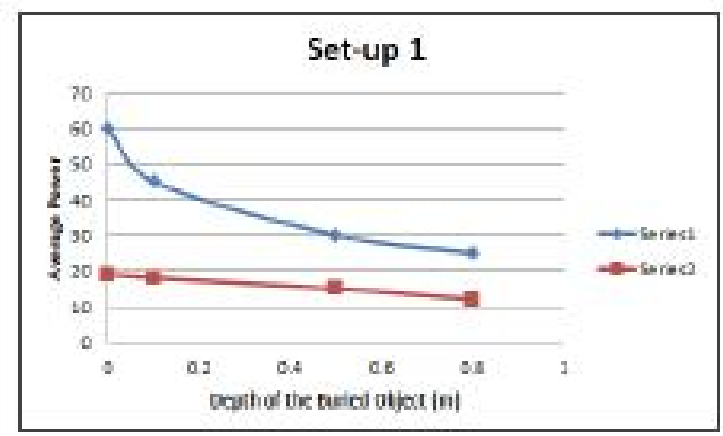

Figure 9: Depth over Amplitude Result

Figure 10 shows the final set up throughout the data gathering based on the results of calibration and testing, which yield the optimum results, which is set up 1 and implementing the parameters as shown in Figure 11.

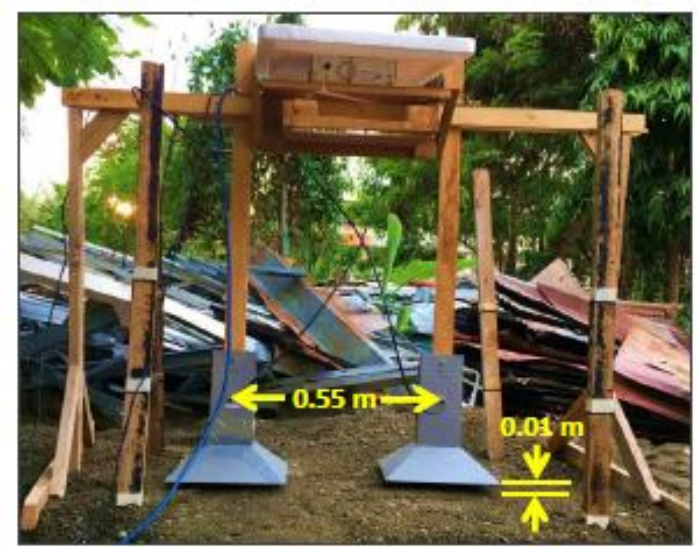

Figure 10: Optimum set up

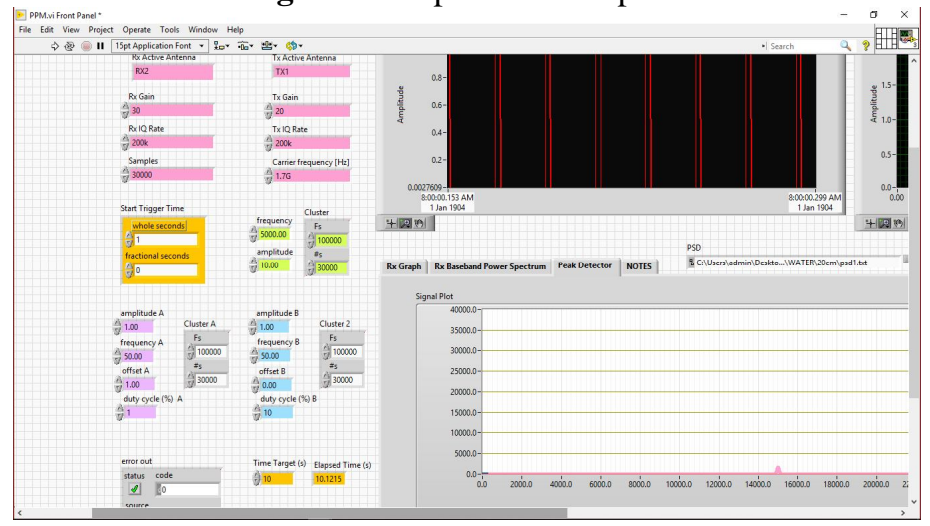

Figure 11: Parameters of GUI

\section{RESULTS}

Based on the setup shown in Figure 10 from the calibration and the optimum parameters, as shown in Figure 11 in the graphical user interface (GUI). The medium is dry sandy soil because of the actual value of the dielectric property, and three (3) targets experimented, namely: metal, plastic, and water with varying depth. In each experiment, fifteen (15) data samples were gathered for the received time-domain signal and its power spectral density. The results are shown in the graphs below. In addition, the power spectral density (PSD) spectrum of one sample will be shown for each experiment.

\subsection{Metal}

Metal is buried at four varying depths as shown in Figure 12. The power spectral density (PSD) of each sample is shown in Figure 13. It can be observed that the power increases with the presence of metal and decreases as the depth increases from $0 \mathrm{~m}$ to $0.6 \mathrm{~m}$. 


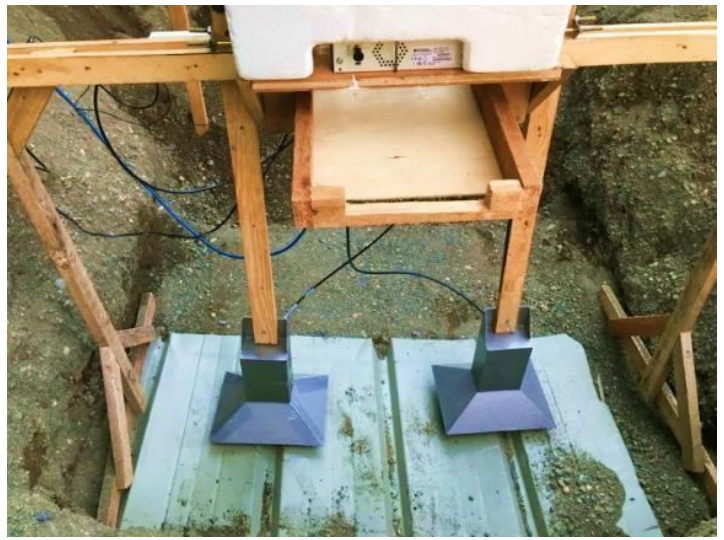

Figure 12: Buried Metal

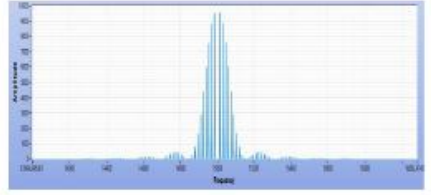

(a) $0.6 \mathrm{~m}$

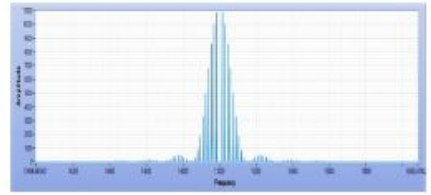

(c) $0.2 \mathrm{~m}$

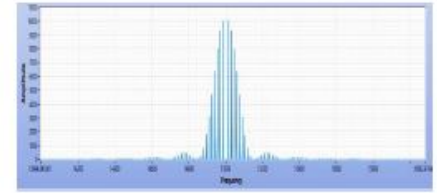

(b) $0.4 \mathrm{~m}$

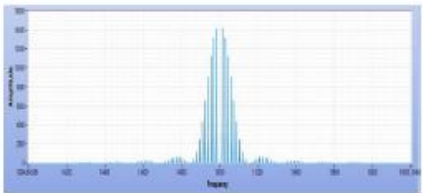

(d) $0 \mathrm{~m}$
Figure 13: Power Spectral Density of Metal (Target)

\subsection{Plastic}

The next target object was plastic, as shown in Figure 14. Again, it was buried at four varying depths. The trend of the average maximum power from the power spectral density (PSD) graph is shown in Figure 15. It can be observed in the graph that the power increases with the presence of plastic and decreases as the depth increases from $0 \mathrm{~m}$ to $0.6 \mathrm{~m}$. The change is more gradual than metal since plastic has a smaller dielectric constant than metal.

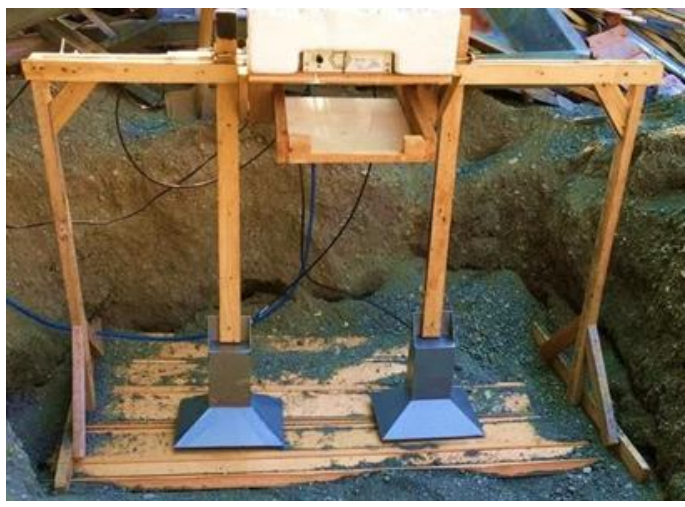

Figure 14: Buried Plastic

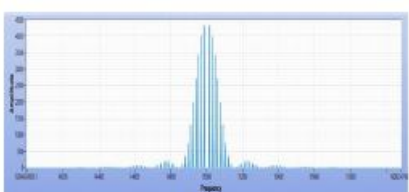

(a) $0.6 \mathrm{~m}$

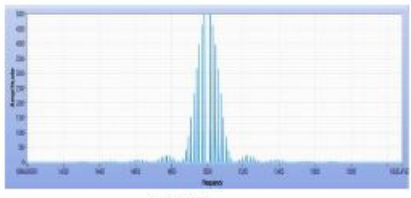

(c) $0.2 \mathrm{~m}$

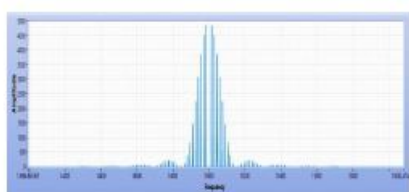

(b) $0.4 \mathrm{~m}$

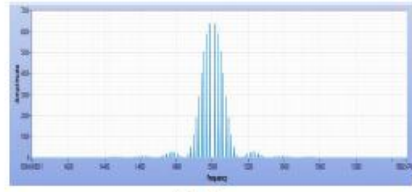

(d) $0 \mathrm{~m}$
Figure 15: Power Spectral Density of Plastic (Target)

\subsection{Water}

The last target object was water, as shown in Figure 16, and it is encapsulated with the Styrofoam box. Again, it was buried along with its container, which is a Styrofoam box since this material has close to air dielectric value at four varying depth. The trend of the average maximum power from the PSD graph is shown in Figure 17. It can be observed in the graph that the power increases with the presence of water at $0.2 \mathrm{~m}$ and $0 \mathrm{~m}$ (which is water itself) but decreases at $0.4 \mathrm{~m}$ and $0.6 \mathrm{~m}$, but, still decreases as the depth increases from $0 \mathrm{~m}$ to $0.6 \mathrm{~m}$. It can be interpreted that the signal attenuates significantly at greater depth.

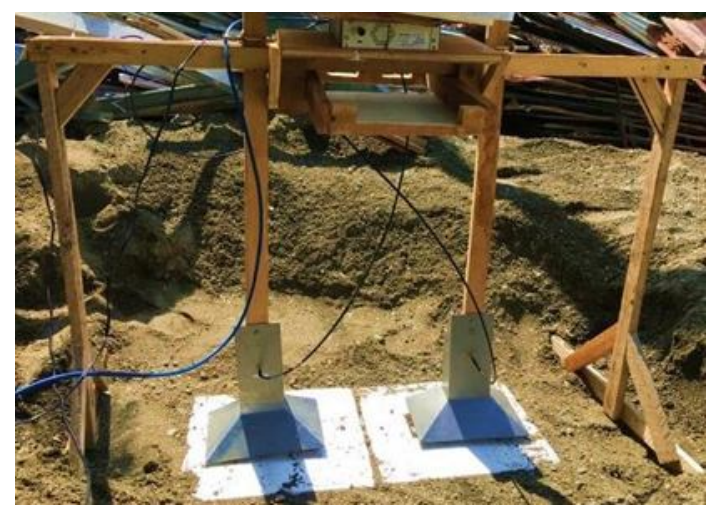

Figure 16: Buried Water

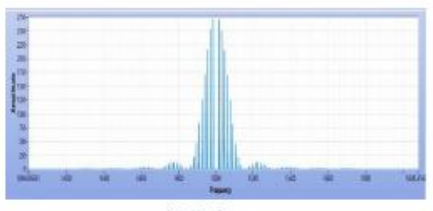

(a) $0.6 \mathrm{~m}$

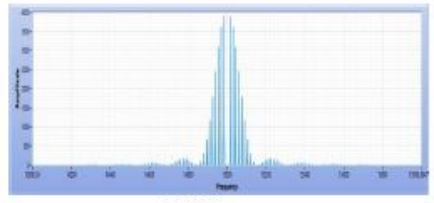

(c) $0.2 \mathrm{~m}$

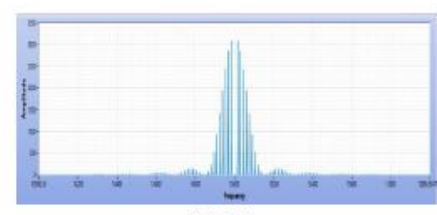

(b) $0.4 \mathrm{~m}$

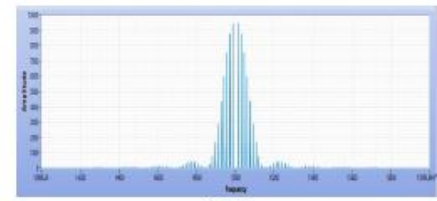

(d) $0 \mathrm{~m}$
Figure 17: Power Spectral Density of Water (Target)

As shown in Figure 18, the behavior of each target with respect to Average Maximum Power with the variation of deepness. It shows it has different unique results which prove 
that the design electromagnetic wave (EM) radar implemented in software-defined radio can detect a subsurface anomaly.

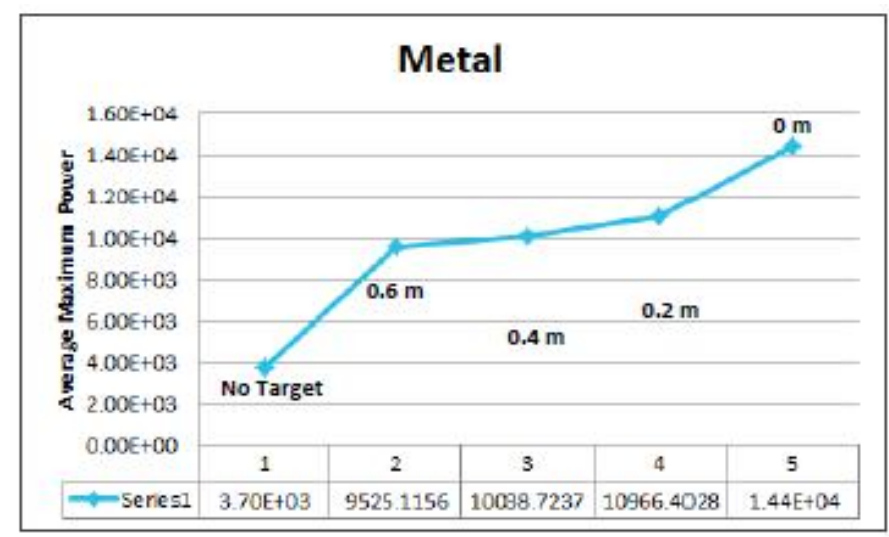

Figure 18a: Trend of Results

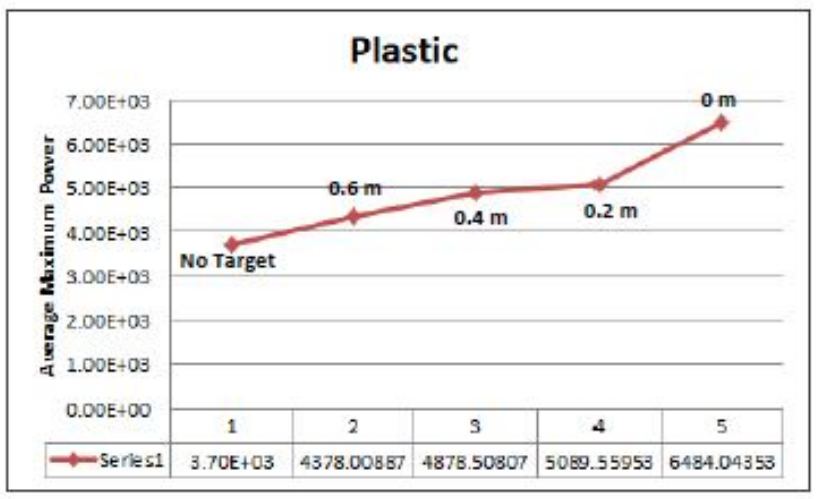

Figure 18b: Trend of Results

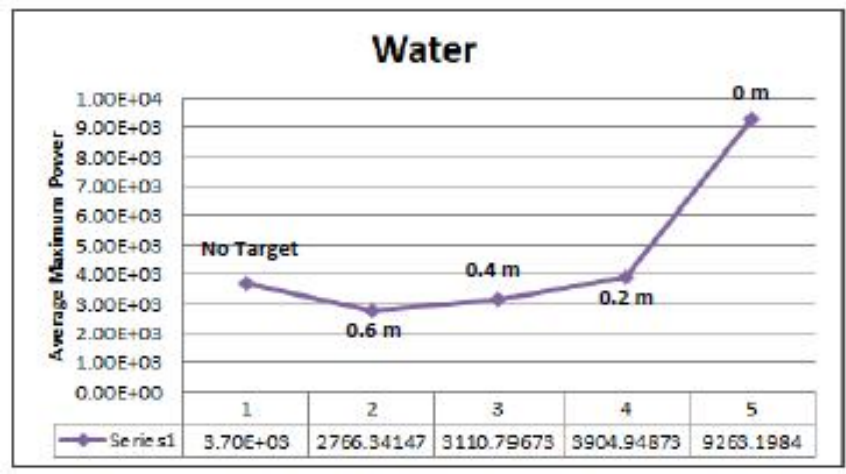

Figure 18c: Trend of Results

\section{CONCLUSION}

The results presented showed that the obtained power spectrum of each material decrease as the depth increases. And comparing the power spectrum of each material, metal has the highest power spectral density. This tendency occurred for each material that possesses a different dielectric constant. The penetration of the signal is also limited since the optimum frequency based on the experiment is $1.7 \mathrm{GHz}$. For deep subsurface detection, it is more convenient to utilize lower to $200 \mathrm{MHz}$ centre frequency and an ultra-wideband antenna that can operate with this low frequency.

\section{REFERENCES}

1. S D. Daniels, "GPR Design Challenges." Accessed: May 28, 2019. [Online]. Available: https://www.academia.edu/11438018/GPR_Design_Cha llenges.

2. S. S.-T. J. of P. Science and undefined 2007, "Applications of Ground Penetrating Radar (GPR) in Detection of Behavior of Groundwater Table Near Pumping Well," iasj.net, Accessed: Feb. 11, 2019. [Online]. Available: https://www.iasj.net/iasj?func=article \&aId=38781.J

3. J. M. Elson and J. M. Bennett, "Calculation of the power spectral density from surface profile data," Appl. Opt., vol. 34, no. 1, p. 201, Jan. 1995, doi: 10.1364/AO.34.000201.

4. W. Wang, L. Hao, X. Li, and J. Sun, "Signal processing technology and its application research of ground penetrating radar (GPR)," IOP Conf. Ser. Earth Environ. Sci., vol. 69, p. 012077, Jun. 2017, doi: 10.1088/1755-1315/69/1/012077.

5. A. Langman and M. R. Inggs, "Pulse versus stepped frequency continuous wave modulation for ground penetrating radar," in IGARSS 2001. Scanning the Present and Resolving the Future. Proceedings. IEEE 2001 International Geoscience and Remote Sensing Symposium (Cat. No.01CH37217), vol. 3, pp. 1533-1535, doi: 10.1109/IGARSS.2001.976902.

6. "FFT Power Spectrum and PSD VI - LabVIEW 2018 Help - National Instruments." https://zone.ni.com/reference/en-XX/help/371361R-01/1 vwave/fft_power_spec_psd/ (accessed Jul. 12, 2020).

7. "FFT and PSD - normalize values - MATLAB Answers - MATLAB Central."

https://au.mathworks.com/matlabcentral/answers/52350 0-fft-and-psd-normalize-values?s_tid=answers_rc1-1_p 1_Topic (accessed Jul. 12, 2020).

8. I.B. Movchan and A.A. Yakovleva, Wave Analogies in the Quantitative Interpretation of Potentila Fields, International Journal of Advanced Trends in Computer Science and Engineering, Vol.9.No.2, March-April 2020, http://doi.rog/10.30534/ijatcse/2020/136922020

9. S. Bohdan, B. Andrii, V. V. Viktor, S. Serhii, H. Halyna, K. Myroslava, and S. Marat, The Electromagnetic Waves Scattering Evaluation on the Composite Material Fractal Structure with Radioisotope Elements, International Journal of Advanced Trends in Computer Science and Engineering, Vol.8.No.5, September-October 2019, http://doi.rog/10.30534/ijatcse/2019/648522019 